\title{
Cannabis and Cannabinoids: From Synapse to Society
}

Neuropsychopharmacology Reviews (20 I8) 43, I-3. doi:I0.1038/npp.2017.255

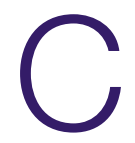
annabis has had a rich and varied history for millennia, across cultures around the world, including ancient China where it was integrated into the pharmacopeia with other plant-based products, to Indian, Persian, and Arabian societies, where it was used in the context of religious rituals or ceremonies (Mechoulam et al, 2014). Evidence of cannabis use from a scientific perspective in the nineteenth century largely began with William O'Shaughnessy, an Irish physician working in India, who studied cannabis for the treatment of rheumatic pain and infantile seizures. Despite the potential therapeutic utility, laws were enacted in the early twentieth century to regulate cannabis cultivation, possession, and use, which significantly changed the scientific framework with which it could be properly studied. The administrative and legal hurdles to study cannabis persist to this day and have restricted comprehensive scientific study of the plant and its constituents, even as scientific understanding of cannabis has exploded.

There have been several major discoveries that have propelled this field forward. The isolation and characterization of delta-9-tetrahydrocannabinol (THC) as the primary psychoactive constituent by Gaoni and Mechoulam (1964) was a significant advance in the field and afforded scientists the ability to study the impact of this molecule in isolation on a variety of physiological and neurobehavioral outcomes. In 1988, Allyn Howlett's lab was the first to demonstrate that cannabinoids exerted their effects on cellular function through activation of what appeared to be a specific G protein-coupled receptor (Devane et al, 1988). The cloning (Matsuda et al, 1990) and localization (Herkenham et al, 1990) of this receptor, termed the cannabinoid type $1\left(\mathrm{CB}_{1}\right)$ receptor, occurred in tandem in 1990 by the labs of Tom Bonner and Miles Herkenham, both of whom worked at the National Institutes of Health. The Mechoulam lab continued to drive the field forward by identifying the first endocannabinoid, a conjugate of arachidonic acid and ethanolamine that was named 'anandamide' from the Sanskrit word 'ananda' meaning bliss (Devane et al, 1992). The 1990s were a boom period in cannabinoid biochemistry, as in 1993 a second cannabinoid receptor $\left(\mathrm{CB}_{2}\right)$ was cloned and characterized as primarily a receptor found on immune cells (Munro et al, 1993). Soon after in 1995, the Sugiura lab (Sugiura et al, 1995), as well as the Mechoulam lab again (Mechoulam et al, 1995), discovered a second endocannabinoid cannabinoid ligand, 2-arachidonoylglycerol (2-AG).
Pioneering work from Cravatt and Piomelli then characterized the enzymes responsible for inactivation of AEA (fatty acid amide hydrolase; Cravatt et al, 1996) and 2-AG (monoacylglycerol lipase; Dinh et al, 2002), respectively. Finally, the major breakthrough that endocannabinoids are released by post-synaptic neurons and act as retrograde synaptic signals, which regulate neurotransmission was published by Wilson and Nicoll (2001) just after the turn of the century. As such, the current state of knowledge about the biology of cannabinoids and endocannabinoids has markedly advanced in the past two decades and the mechanisms by which these molecules can influence a host of physiological processes has been well characterized. We are currently at an interesting crux in history, as the legal status of cannabis is changing markedly throughout individual states in the USA, federally within Canada as well as in some western European countries (such as Portugal), highlighting the timeliness of this issue of Neuropsychopharmacology Reviews. Given the broad impact of cannabis and cannabinoids, this issue encompasses a wide array of articles that discuss the current state of knowledge of cannabis and cannabinoids from synapses to society and everything in between.

Starting at the synapse, the first article in this issue by Busquets-Garcia and colleagues, provides a comprehensive discussion about the complexity through which cannabinoids and endocannabinoids signal at the $\mathrm{CB}_{1}$ receptor. Gone are the days where we have conceptualized endocannabinoids as nothing more than a 'circuit breaker' where elevations in neuronal excitability trigger the release of endocannabinoids to dampen presynaptic drive onto a cell. In fact, it is now widely recognized that $\mathrm{CB}_{1}$ receptors are expressed on a host of cell types throughout the brain, and that activation of $\mathrm{CB}_{1}$ receptors on neurons can have profoundly different effects than when $\mathrm{CB}_{1}$ receptors are activated on astrocytes. In fact, even within a cell, the localization of $\mathrm{CB}_{1}$ receptors to either the membrane of the axon terminal versus intracellular mitochondria can have very different effects on cellular processes. These complexities, as well as much more, are discussed in depth in this article (Busquets-Garcia et al, 2017).

At a developmental level, a growing body of evidence has also indicated that endocannabinoid signaling is critical for the development, maturation, and sculpting of neural circuits. While research in this area has typically focused on embryonic and early development of the nervous system, 
recent findings indicate that endocannabinoids continue to impact brain development throughout adolescence. In the next article, Meyer et al (2017) review findings implicating a fundamental role of endocannabinoid signaling in the development of cortico-limbic circuits during adolescence and how disruptions of this system could modulate normative developmental trajectories of brain maturation. More so, this review highlights recent translational work in rodents and humans, leveraging genetic variance in endocannabinoid signaling, as an approach to understand how endocannabinoids modulate adolescent brain development.

Another issue of relevance for understanding the biological effect of cannabis and cannabinoids is the influence of sex, which is the focus of the next article in this issue (Cooper and Craft, 2017). Sex differences are a factor long overlooked in both preclinical and clinical science, but that has relevance to both therapeutic applications and cannabis abuse. Although men have higher rates of recreational cannabis use and cannabis use disorder than women, use of cannabis for its potential therapeutic effects tends to be equal as a function of sex. This review provides a translational perspective on the sex-dependent effects of cannabis and cannabinoids by synthesizing findings from preclinical and clinical studies focused on sex comparisons of their therapeutic potential and abuse liability, two specific areas that are of significant public health relevance.

The next series of reviews focus on specific processes that cannabinoids and endocannabinoids are known to influence. A highly discussed area in the realm of cannabis and cannabinoid therapeutics is pain. The recent marked rise in rates of opioid overdoses and the subsequent tightening of guidelines for prescribing opioid medications, concurrent with legalized cannabis for medical use in many locales highlight the timeliness of a review of how endocannabinoids and phytocannabinoids produce antinociceptive effects in inflammatory and neuropathic rodent pain models, including a discussion of whether cannabinoids have opioidsparing effects (Donvito et al, 2017). Following this, another area that has generated a lot of discussion of the potential therapeutic use of cannabis and cannabinoids is posttraumatic stress disorder (PTSD). In the article by Hill et al (2017) the role that endocannabinoids have in the processing of threatening and emotionally salient information, anxiety, and the consolidation of emotional memories provides a biological basis for the potential therapeutic use of cannabinoids to reduce hyper-vigilance, improve extinction to trauma-paired cues, and promote sleep in patients with PTSD. The authors note that as is the case for cannabis and the treatment of pain, high-quality data are critically need to evaluate the efficacy of endocannabinoid modulation or cannabis-related medications to treat PTSD.

The next two articles in this issue focus on aspects of cannabinoids and endocannabinoid that relate to reward processes and addiction. The first of these articles by Wenzel and Cheer (2017) focuses heavily on the mechanisms by which endocannabinoids interact with neural signaling systems in the brain that are well established to regulate rewarding processes, particularly the dopaminergic and opioidergic systems. This review focuses on how endocannabinoids can modulate both dopamine and endogenous opioid release and signaling to discuss how endocannabinoid signaling may naturally sculpt reinforcement and reward (Wenzel and Cheer, 2017). Outside of the role of endocannabinoids, however, cannabis itself is also a drug of abuse, and preclinical models of drug reward and reinforcement can be used to investigate the neurobiology of these effects. This area is reviewed by Panlilio and Justinova (2017) in the next article. Although the primary psychoactive component of cannabis, THC, is not robustly self-administered in most preclinical models, the rewarding and reinforcing effects of other cannabinoid agonists have been well characterized and offer insight into addiction-related brain functioning and behavior. Preclinical studies can also assess the direct influence of cannabinoid exposure on the reinforcing effects of other drugs of abuse and vice versa, while avoiding the confounds inherent to epidemiological studies reporting an association between early cannabis use and subsequent use of other drugs (Panlilio and Justinova, 2017).

One of the other issues regarding cannabis that is in the popular media regularly is the balance of the constituents of cannabis and how they interact. While THC is known to be the primary psychoactive component in cannabis, in recent years the phytocannabinoid, cannabidiol (CBD), has generated enormous interest in both the scientific and public sphere. CBD appears to lack the psychoactivity associated with THC, but may modulate certain of THC's effects. At a functional level, $\mathrm{CBD}$ has little direct activity at the cannabinoid receptors, but appears to influence the activity of a range of neurochemical systems and receptor subtypes (eg, serotonin and TRPV1). The focus of the next review in this issue is to evaluate preclinical and clinical findings regarding the pharmacology and behavioral interactions between THC and CBD, particularly as it pertains to anxiety, psychosis, and cognition (Boggs et al, 2017).

Moving more into the clinical realm, the next article in this issue reviews the current state of knowledge regarding endocannabinoids in humans, how we measure them, what regulates their release, and their relevance to psychiatric illness (Hillard, 2017). Specifically, as most studies in humans measure circulating levels of endocannabinoids, this review summarizes what we know about circulating endocannabinoids, particularly with respect to what cell types may contribute to their production and whether measuring endocannabinoid levels in the blood has relevance to endocannabinoid function in the brain. As alterations in circulating endocannabinoids have been identified in several psychiatric conditions, this article is an important primer to help interpret plasma endocannabinoid levels and design future studies appropriately.

Finally, the last two reviews in this issue focus largely on issues of public health and epidemiology. Cannabis use disorder is on the rise among adults, and a significant subset of individuals seeking treatment for their drug use cite cannabis as their primary drug. Yet CUD remains 
undertreated, and the literature on randomized controlled clinical trials testing pharmacotherapies for the treatment of CUD is sparse when compared to other commonly abused drugs. The authors of this review provide a comprehensive, up-to-date summary of the field to date, while also providing an essential first-hand perspective about the unique issues encountered while treating this disorder (Brezing and Levin, 2017). Finally, there are vast societal changes in attitudes toward cannabis, and the legality of cannabis for recreational and medical use, but little discussion in the public sphere on the implications of these changes. The review by Hasin, 2018 provides an overview of the epidemiology of cannabis use, including issues such as prenatal cannabis exposure, incidence of CUD, cannabis-related emergency room visits, as well as the evidence of reduced opioid and psychiatric medication use in states that have legalized the medical use of cannabis. This evidence-based discussion of the positive and negative effects associated with cannabis use, with careful consideration of potential confounds in interpreting these associations, can inform the public, health professionals, and policy makers on as polarizing a topic as cannabis.

Taken together, we hope that the collection of reviews in this article will act as a primer to anyone in the field as to the impact of cannabinoids, from synapses up to policy. The information presented represents the current state of knowledge across a wide array of areas of science and we hope that this issue acts as a point of intersection for scientists from varying backgrounds to broaden their horizons, and to develop new perspectives and scientific questions about cannabinoids and cannabis. At the risk of being too tongue-in-cheek, we believe that it's high time that the smoke is cleared in this field about the actual science and evidence surrounding cannabis and cannabinoids, and we believe that this issue of Neuropsychopharmacology Reviews represents an ideal source for the dissemination, and future discussion, of this information.

\section{FUNDING AND DISCLOSURE}

The authors declare no conflict of interest.

\section{REFERENCES}

Boggs DL, Nguyen JD, Morgenson D, Taffe MA, Ranganathan M (2018). Clinical and pre-clinical evidence for functional interactions of cannabidiol and $\Delta 9$-tetrahydrocannabinol. Neuropsychopharmacology 43: 142-154.

Brezing CA, Levin FR (2018). The current state of pharmacological treatments for cannabis use disorder and withdrawal. Neuropsychopharmacology 43: 173-194.

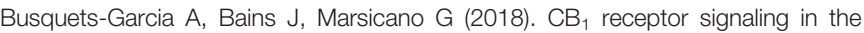
brain: extracting specificity from ubiquity. Neuropsychopharmacology 43: 4-20.
Cooper ZD, Craft RM (2018). Sex-dependent effects of cannabis and cannabinoids: a translational perspective. Neuropsychopharmacology 43: 34-51.

Cravatt BF, Giang DK, Mayfield SP, Boger DL, Lerner RA, Giula NB (1996). Molecular characterization of an enzyme that degrades neuromodulatory fattyacid amides. Nature 384 : 83-87.

Devane WA, Dysarz FA, Johnson MR, Melvin LS, Howlett AC (1988). Determination and characterization of a cannabinoid receptor in rat brain. Mol Pharmacol 34: 605-613.

Devane WA, Hanus L, Breuer A, Pertwee RG, Stevenson LA, Griffin G et al (1992). Isolation and structure of a brain constituent that binds to the cannabinoid receptor. Science 258: 1946-1949.

Dinh TP, Carpenter D, Leslie FM, Freund TF, Katona I, Sensi SL et al (2002). Brain monoglyceride lipase participating in endocannabinoid inactivation. Proc Natl Acad Sci USA 99: 10819-10824.

Donvito G, Nass SR, Wilkerson JL, Curry ZA, Schurman LD, Kinsey SG et al (2018). The endogenous cannabinoid system: a budding source of targets for treating inflammatory and neuropathic pain. Neuropsychopharmacology 43: 52-79.

Gaoni Y, Mechoulam R (1964). Isolation, structure and partial synthesis of an active constituent of hashish. J Am Chem Soc 86: 1646-1647.

Hasin DS (2018). U.S. Epidemeology of Cannabis Use and Associated Problems. Neuropsychopharmacology 43: 195-212.

Herkenham M, Lynn AB, Little MD, Johnson MR, Melvin LS, de Costa BR et al (1990). Cannabinoid receptor localization in brain. Proc Natl Acad Sci USA 87: 1932-1936.

Hill MN, Campolongo P, Yehuda R, Patel S (2018). Integrating endocannabinoid signaling and cannabinoids into the biology and treatment of posttraumatic stress disorder. Neuropsychopharmacology 43: 80-102.

Hillard CJ (2018). Circulating endocannabinoids: from whence do they come and where are they going? Neuropsychopharmacology. 43: 155-172

Matsuda LA, Lolait SJ, Brownstein MJ, Young AC, Bonner TI (1990). Structure of a cannabinoid receptor and functional expression of the cloned cDNA. Nature 346: 561-564.

Mechoulam R, Ben-Shabat S, Hanus L, Ligumsky M, Kaminski NE, Schatz AR et al (1995). Identification of an endogenous 2-monoglyceride, present in the cainine gut, that binds to cannabinoid receptors. Biochem Pharmacol 50: 83-90.

Mechoulam R, Hanus LO, Pertwee R, Howlett AC (2014). Early phytocannabinoid chemistry to endocannabinoids and beyond. Nat Rev Neurosci 15: 757-764.

Meyer HC, Lee FS, Gee DG (2018). The role of the endocannabinoid system and genetic variation in adolescent brain development. Neuropsychopharmacology 43: 21-33.

Munro S, Thomas KL, Abu-Shaar M (1993). Molecular characterization of a peripheral receptor for cannabinoids. Nature 365: 61-65.

Panlilio LV, Justinova Z (2018). Preclinical studies of cannabinoid reward, treatments for cannabis use disorder and addiction-related effects of cannabinoid exposure. Neuropsychopharmacology 43: 116-141.

Sugiura T, Kondo S, Sukagawa A, Nakane S, Shinoda A, Itoh K et al (1995). 2Arachidonoylglycerol: a possible endogenous cannabinoid receptor ligand in brain. Biochem Biophys Res Commun 215: 89-97.

Wenzel JM, Cheer JF (2018). Endocannabinoid regulation of reward and reinforcement through interaction with dopamine and endogenous opioid signaling. Neuropsychopharmacology 43: 103-115.

Wilson RI, Nicoll RA (2001). Endogenous cannabinoids mediate retrograde signaling at hippocampal synapses. Nature 410: 588-592.

Margaret Haney ${ }^{*, 1}$ and Matthew N Hill ${ }^{2}$ ${ }^{1}$ Department of Psychiatry, Columbia University Medical Center and the New York State Psychiatric Institute, New York, NY, USA; ${ }^{2}$ Departments of Cell Biology and Anatomy \& Psychiatry, Hotchkiss Brain Institute, Cumming School of Medicine, University of Calgary, Calgary AB, Canada E-mail:mh235@cumc.columbia.edu 